\title{
Utilidad de la historia médico-psicológica en estudiantes que inician ciclos clínicos en la carrera de medicina como instrumento para la comprensión integral del paciente y facilitador de la alianza terapéutica
}

Juan A. Mejías-Vizcarro, Lizbeth Beltrán-Hernández

Introducción. La formación del estudiante de medicina busca la adquisición de conocimientos teóricos y prácticos, actitudes ético-morales, habilidades y destrezas en la comunicación interpersonal. Tener una formación integral permitirá establecer una relación médico-paciente adecuada para ofrecer una mejor atención desde el primer contacto que tienen con él.

Sujetos y métodos. Se implementó y evaluó una experiencia vivencial en la que los estudiantes realizaron historias clínicas de tipo médico-psicológico a pacientes del hospital donde llevan a cabo sus rotaciones, sin que se encontraran presentes los profesores, agregando un comentario que reflejaba lo que experimentaron en el encuentro. Se utilizó una metodología cualitativa de tipo investigación-acción participativa. Posteriormente se analizaron los comentarios de cada estudiante y se compararon con lo encontrado en la bibliografía.

Resultados. Ante esta experiencia, la mayoría de los estudiantes se sintieron ansiosos o incómodos al indagar en temas personales como la sexualidad. Así mismo, sintieron empatía ante la enfermedad o la historia personal de cada paciente, lo que les hizo entender mejor su manera de ser, su situación y su manera de afrontarla, y comprender cómo la relación médico-paciente influye en el paciente y en su enfermedad.

Conclusión. La realización de este tipo de historia clínica por parte de los estudiantes al inicio de su formación clínica es una experiencia vivencial que deja un aprendizaje con un importante impacto emocional, pues le permite el contacto directo con el ser humano enfermo y no solamente con la enfermedad, fortaleciendo la relación médico-paciente.

Palabras clave. Estudiantes de medicina. Relación médico-paciente.

The utility of medical-psychological history in medical students who start rotations as a way to comprehend the patient and as a facilitator for the therapeutic alliance

Introduction. Medical students' formation needs the acquisition of theoretical and practical knowledge, ethical-moral attitudes, and interpersonal communication skills. Having a comprehensive education will establish an adequate doctorpatient relationship to offer better care since the first contact they have with them.

Subjects and methods. A qualitative methodology of participatory research-action type was used. Medical students who were beginning their clinical practice applied medical-psychological histories to patients of the hospital where they carry out their clerkships, without the teachers being present, adding a comment that reflects what they experienced after having the encounter. Subsequently, the comments of each student were analyzed and compared with what was found in the literature.

Results. Most of the students felt anxious or uncomfortable when they inquired about personal topics such as sexuality. They also felt empathy for the illness and/or personal history of each patient, which helped them understand their way of being, their situation, as well as their way of dealing with it and comprehend how the doctor-patient relationship influences the patient and in their illness.

Conclusion. Carrying out this type of medical history by the students who start their clinical training is an experience that impacts importantly on an emotional level, because it allows direct contact with the sick human being and not only with the disease, strengthening the doctor-patient relationship.

Key words. Doctor-patient relationship. Medical students.
Facultad de Medicina. Universidad Nacional Autónoma de México. México DF, México.

Correspondencia:

Dr. Juan Antonio Mejías Vizcarro. Departamento de Psiquiatría y Salud Mental. Facultad de Medicina. Universidad Nacional Autónoma de México. Ciudad Universitaria. Circuito Interior y Cerro del Agua Facultad de Medicina, edificio F. Avda. Universidad 3000. 04510 Coyoacán. México DF, México.

E-mail:

jamejviz@yahoo.com

Recibido: 28.09.18.

\section{Aceptado:}

11.10.18.

Conflicto de intereses: No declarado.

Competing interests: None declared.

(c) 2019 FEM

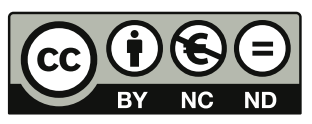

Artículo open access bajo la licencia CC BY-NC-ND (https:// creativecommons.org/licenses/ by-nc-nd/4.0/).

ISSN: 2014-9832

ISSN (ed. digital): 2014-9840 


\section{Introducción}

Es evidente que el ejercicio de la medicina actual se ha caracterizado por una creciente especialización, aunado al progreso tecnológico y científico, lo que ha incrementado los costes y ha llevado a que el paciente tenga más oportunidades en cuanto a un diagnóstico y tratamiento certeros. Sin embargo, ha propiciado que sea menos acompañado en su realidad personal por el médico, lo que provoca que la relación médico-paciente clásica, basada en la confianza y la comprensión del significado de la enfermedad, se haya vuelto cada vez más impersonal y contractual.

Diversas facultades de medicina en el mundo consideran conveniente que los estudiantes de la carrera tengan una formación más integral y, además de adquirir conocimientos clínicos de tipo médico-científico, se les proporcione un entrenamiento que enfatice el aprendizaje de valores, actitudes ético-morales, habilidades y destrezas comunicacionales interpersonales, así como la adquisición de competencias, como lo propuesto por el comité de graduados de educación médica (ACGME) en Estados Unidos [1].

El propósito de muchas facultades en la enseñanza médica es formar médicos de excelencia científica y técnica, tratando de unirlo a una sólida formación ética y compromiso de servicio. Para lograrlo, algunas escuelas han introducido en el currículo cursos de bioética, psicología, comunicación, antropología y otras disciplinas de humanidades médicas. Sin embargo, estos cursos teóricos parecen insuficientes para que los estudiantes lleguen a comprender el significado de la enfermedad para el paciente y su entorno sociofamiliar, es decir, les falta ser testigos directos de la enfermedad de los pacientes a quienes conozcan en lo personal. Como esto difícilmente se logra en la enseñanza de pregrado, parece necesario crear y probar formas de hacerlo. La bibliografía muestra experiencias no evaluadas de acompañamiento prolongado de enfermos en el posgrado [1] y propone incorporar precozmente a los estudiantes a la práctica clínica con formas variadas de participación [2].

Se ha demostrado que, aunque los estudiantes suelen tener dificultades en sus primeros encuentros con los pacientes, rara vez lo comentan con sus docentes, y que quienes reciben formación en comunicación demuestran mayor comprensión y competencia en decisiones compartidas [3-5]. Por otra parte, la participación de los pacientes como 'educadores' tiene un beneficio para que los estudiantes mejoren sus actitudes hacia ellos [6]. No obstante, no se han evaluado de manera directa los cambios que se producirían en estudiantes sometidos a una experiencia vivencial con un 'paciente tutor' previamente seleccionado, que trasmita el significado profundo de vivir y padecer una enfermedad desde su perspectiva y la de su entorno familiar.

Una forma sería que los estudiantes realicen historias clínicas médico-psicológicas que permitan tener un contacto más personal y profundo al interrogar al paciente no solamente sobre los aspectos relacionados con su padecimiento y su tratamiento técnico y científico, sino también sobre otros aspectos de su vida que, por contener información más extensa y personal, requieren al menos dos o tres sesiones; esto permite y obliga a tener una interacción mayor entre el estudiante de medicina que cursa sus ciclos clínicos e inicia sus primeros encuentros y el ser humano enfermo, ofreciendo una visión diferente a la que permite el abordaje técnicocientífico y dando mayor significado a la relación médico-paciente.

Un antecedente al respecto es el estudio que realizaron Ortiz et al [6], quienes implementaron una experiencia vivencial en la cual estudiantes de tercer año de medicina se constituyeron en testigos y acompañantes de un enfermo por un período de dos meses, manteniendo uno o dos encuentros semanales con él y con su familia. Fueron evaluados al inicio y un mes después con una metodología cualitativa, con la técnica de grupo focal mediante dos entrevistas dirigidas por uno de los autores. Al término de cada encuentro, cada estudiante escribió una narración sobre sus reflexiones personales y datos de la historia del paciente. Se concluyó que los participantes tuvieron una experiencia importante: no sólo conocieron el sufrimiento de algunos pacientes, sino que, en otros casos, observaron cómo se pueden adaptar a las condiciones que les exige la enfermedad. A su vez, vivieron la experiencia de entregar apoyo y afecto al paciente sólo a cambio de una satisfacción personal. Esta experiencia posibilitó al estudiante comprender que la enfermedad ocurre de manera diferente en cada persona y que adquiere consecuencias y significados propios en cada caso, tanto para el paciente como para su familia.

En otro estudio, Beca et al [7] destacan que, en los hospitales donde se realiza docencia e investigación, se establece una relación entre los pacientes y los estudiantes de medicina, ya que la atención se realiza por docentes clínicos acompañados de alumnos que observan o efectúan algunas de estas maniobras. Los pacientes exponen así su historia clínica y su cuerpo y pasan a ser alguien que, además de recibir una atención, es también objeto de aprendi- 
zaje. En estos hospitales se acepta casi sin dudar que los pacientes constituyan un recurso para la docencia $[8,9]$, siendo pocos los que se cuestionan si se necesita autorización previa.

Por otra parte, la relación humana entre pacientes y estudiantes se ha estudiado poco $[10,11]$, por lo que se realizó un estudio cualitativo indagando la opinión que tienen los pacientes sobre la atención que reciben por parte de los estudiantes de medicina. Este tipo de información permitió conocer la disposición de los enfermos a colaborar en la enseñanza de pregrado, su opinión respecto a ella y, a la vez, determinar las diferentes visiones que pueden existir entre pacientes de un hospital público y uno privado. Los entrevistados del hospital público manifestaron el agrado de ser atendidos por alumnos de medicina. Aceptaron ser abordados por estudiantes, ya que éstos les dedican más tiempo, lo que les permite aclarar dudas e inquietudes, pero señalaron que aunque su carácter jovial es un elemento favorable, prefieren que su atención se limite al examen físico y la historia clínica, de manera que su intervención no sea decisiva en diagnósticos o tratamientos. Manifestaron también que les era indiferente que el alumno fuera hombre o mujer, que reconocen la atención de los estudiantes como una necesidad para su aprendizaje y piensan que en los centros privados se les negaría esta posibilidad. Los entrevistados de la clínica privada, en cambio, manifestaron no tener problemas en ser observados por un estudiante, pero idealmente no en grupo, sino un estudiante con el médico. Tampoco consideran que los estudiantes deban atenderles, cuestionando su idoneidad para formular diagnósticos y tratamientos. La única atención de estudiantes que permitirían sería la realización de entrevistas, examen físico, curaciones o procedimientos menores. Aseguraron conocer la posibilidad de negarse a ser atendidos por estudiantes y, en general, rechazarían su atención si antes no se les pide autorización cuando los estudiantes tienen muy pocos años de estudio, cuando se muestran muy inseguros, prepotentes o con mala presencia, o ante una enfermedad crítica. La disposición menos favorable hacia los estudiantes se relacionó con desconocimiento sobre su rol y con el temor de errores diagnósticos.

Beca et al [12] investigaron la relación entre estudiante de medicina y paciente, pero en este caso desde la visión de los estudiantes. Además de la relación de todo el equipo médico, los pacientes y muchas veces su familia tienen un contacto con el estudiante de medicina, por lo que el enfermo pasa de ser no sólo alguien que recibe una atención o prestación, sino también alguien de quien un estudiante apren- de, por lo que no debe ser objeto pasivo de aprendizaje, sino un sujeto voluntario que acepta este rol.

Otros estudios han analizado la respuesta de los estudiantes frente a los pacientes $[11,13]$; según estos autores, es conveniente conocer la visión de los estudiantes para comprender la participación de ambos en este vínculo. Para ello, el propósito de su estudio fue conocer los pensamientos y vivencias cuando se enfrentan con los enfermos, cómo abordar las dificultades o conflictos, cómo influyen en su visión sobre la profesión médica y el concepto que tienen sobre los derechos de los pacientes. El estudio fue realizado por medio de entrevistas aplicadas por los autores a 30 alumnos de tercer año de carrera (15 hombres y 15 mujeres), con preguntas abiertas que se grabaron y posteriormente se transcribieron. Los estudiantes señalaron haber experimentado algunas dificultades o debilidades, como dificultad para iniciar la conversación, falta de empatía, temor a los tutores, incomodidad ante sus compañeros, hacer historias demasiado detalladas, temor a equivocarse, sobreinvolucrarse con el enfermo, dificultad para ponerse en el lugar de una persona más humilde, transmitir su nerviosismo al paciente, miedo a preguntar sobre temas íntimos, sentirse muy jóvenes al lado de los pacientes y no saber afrontar los aspectos emocionales. Otro temor compartido fue 'sobrepasar su rol de estudiante de medicina'. Un problema que afrontaron, aunque de manera infrecuente, fue el rechazo de pacientes que inicialmente no querían ser entrevistados ni examinados por alumnos. En general, esto se debió a cansancio o porque los enfermos ya habían sido entrevistados y examinados reiteradamente. También se presentaron situaciones de pacientes con sordera o dificultad para comunicarse. Cuando se relacionaron con enfermos terminales, muy deprimidos o de extrema gravedad, los estudiantes no supieron cómo afrontar la situación. Algunos señalaron ponerse tan nerviosos que no pudieron realizar su trabajo, especialmente si estaban frente a otros compañeros o a su tutor. El examen físico fue motivo de inhibición, principalmente ante exámenes ginecológicos. Preguntar sobre sexualidad, orientación sexual o consumo de drogas fueron situaciones que generaron gran estrés a algunos estudiantes. También se enfrentaron a pacientes que los trataron mal o que les hicieron sentirse inseguros. Sin embargo, estos problemas fueron excepcionales y los estudiantes los relataron como episodios que ellos mismos pudieron superar. Finalmente, todos los estudiantes manifestaron que sus encuentros con pacientes, contrariamente a sus temores previos, fueron muy gratificantes. Se sintieron impresiona- 
dos por la buena disposición de los pacientes hacia ellos. En general, los estudiantes manifestaron que los pacientes los aprecian, aunque en este tema aparecen diferencias entre estudiantes masculinos y femeninos. Los hombres sentían que daban una impresión de seriedad, mientras que las mujeres, en cambio, se reconocieron más inseguras y debían ganarse la confianza de los pacientes.

Dado que la relación médico-paciente y la aplicación de historias clínicas médico-psicológicas son algunos de los temas impartidos en el tercer año de la carrera de medicina de la Facultad de Medicina de la Universidad Nacional Autónoma de México (UNAM), se implementó la aplicación de estas historias a algunos de sus pacientes. El hecho de presentarse como estudiantes de medicina ofrece un acercamiento diferente a los pases de visita, y el acceso de los estudiantes junto con sus profesores, como se refirió en un inicio, brindaba historias clínicas más profundas y completas que ofrecían a los estudiantes un acercamiento mucho más personal e intenso con los pacientes. Debido a que están cursando el inicio de su formación clínica, ello constituye una experiencia novedosa y de mucho valor para vivir la relación médico-paciente y el padecimiento de este último.

Con este propósito, se diseñó una experiencia de acompañamiento a un paciente evaluando de manera cualitativa la percepción de los estudiantes sobre el significado de la enfermedad y, con ello, demostrar y consolidar la relevancia de la relación médico-paciente.

Además, se ejemplifican algunos de los casos vistos por estudiantes así como el comentario que hicieron, para posteriormente hacer un análisis de este ejercicio. Ello se compara con lo encontrado en la bibliografía sobre las formas de explorar esta situación en un contacto directo con el paciente y, en algunas ocasiones, con sus familiares.

\section{Sujetos y métodos}

Se implementó y evaluó una experiencia vivencial en la que los estudiantes de medicina realizaron historias clínicas a pacientes del hospital donde llevan a cabo sus rotaciones. Las historias clínicas fueron de tipo médico-psicológico, teniendo entre sus apartados que el estudiante hiciera un comentario de esta vivencia, así como los sentimientos y reflexiones que le despertó. Dichas entrevistas las realizan los estudiantes de forma independiente, sin que se encontraran presentes los profesores, consiguiendo la cooperación de los pacientes y sus fami- liares. Después de realizar las historias clínicas médico-psicológicas, los alumnos las transcriben para su supervisión y agregan un comentario bajo el título 'sentimientos y reacciones del entrevistador hacia el paciente. En este comentario se capta la esencia de lo que el estudiante de medicina experimenta al tener el encuentro directo.

Los estudiantes fueron seis alumnos de quinto semestre de la Facultad de Medicina de la UNAM, quienes inician sus rotaciones clínicas y cursan la materia de 'Medicina psicológica y comunicación'. Cada alumno eligió libremente al paciente que deseaba entrevistar, requiriendo de una a tres sesiones para completar el ejercicio. Previamente, los estudiantes recibieron en las clases toda la información de cómo se integra este tipo de historia clínica, sus diferentes apartados, e incluso se llevó a cabo la lectura de ejemplos de algunas historias realizadas por estudiantes de años anteriores. Se contó con el consentimiento oral por parte de los alumnos que accedieron a participar.

Se utilizó una metodología cualitativa de tipo investigación-acción participativa, basada en la idea de que a través de la experiencia vivida se puede comprender la esencia del tema [14]. Los estudiantes relataron y transcribieron el caso al que se enfrentaron, así como las reflexiones y sentimientos que presentaron ante dicha situación. Posteriormente se analizaron los comentarios de cada estudiante y se compararon con lo encontrado en la bibliografía. Por el tipo de estudio, no se requirió autorización por parte del comité de ética.

\section{Resultados}

En general, los estudiantes manifestaron sentirse ansiosos. Otros más, incomodidad en el momento de indagar en temas personales como la sexualidad. Muchos de ellos sintieron empatía ante la enfermedad o la historia personal de cada paciente, lo que los hizo comprender mejor su manera de ser, la situación por la que están pasando y su manera de afrontarla; por ejemplo, comentan: 'conocer a fondo la historia de cada uno es extraordinario porque sabes qué hay más allá de la enfermedad y puedes comenzar a entender muchas cosas', 'te ayuda a relacionarte, a buscar la forma de meterte en la vida del paciente'. Así mismo, esto les ayudó a comprender cómo la relación médico-paciente puede influir en el paciente y en su enfermedad: 'reconfortar al paciente y darle ánimos para seguir con su tratamiento, como debe ser, así ganamos más nosotros porque nos hacemos buenos médicos'. 
Por otro lado, se encontró que la mayoría de los pacientes estuvieron dispuestos e incluso mostraron conformidad y gratitud de ser parte del ejercicio para la formación de los estudiantes, pues refieren que los médicos a cargo no disponen del tiempo o interés suficiente al atenderlos. $\mathrm{Al}$ respecto, comentan: 'su nuera me preguntó el motivo de la entrevista, yo en todo momento le expliqué que era parte de mi formación como médico y me comentó que le daba mucho gusto ya que muchas veces parece que a los médicos no les interesa'. Otro alumno relata: 'durante la entrevista me sentí muy a gusto con la paciente porque fue muy cooperadora, amable, no mostró pena o impaciencia ante la entrevista [...]; la paciente me dio a entender que apreciaba el tiempo que le dedicaba.' Por último, una estudiante cita las palabras de la paciente: 'gracias a ti por escucharme, espero haber sido de ayuda en tu tarea y échale muchas ganas'.

También refirieron tener dificultades y sentirse nerviosos durante los primeros encuentros, pero poco a poco lograron tener más confianza en sí mismos y, principalmente, transmitir esa confianza al paciente, logrando finalmente consolidar una buena relación médico-paciente. Más que nuevos hallazgos, reafirmaron conceptos estudiados con anterioridad y tomaron conciencia de la importancia de comprender globalmente al enfermo y no sólo la enfermedad, como un elemento necesario para ejercer una buena medicina.

A continuación se transcriben los casos y reflexiones de los estudiantes en cada uno de ellos.

\section{Caso 1}

Mujer de 26 años, madre soltera con educación secundaria, que acude al hospital por presentar un cuadro urinario caracterizado por dolor en la zona lumbar, que se irradia a la zona sacra y parte superior de glúteos, así como disuria y hematuria.

La alumna comenta al respecto: 'por momentos me sentía muy incómoda, por ejemplo, cuando relató el tipo de relación que llevaba con su jefe y su primo, porque me parece que después de todo lo que ha vivido busca ocultar la soledad y la tristeza que siente teniendo relaciones sexuales con quien puede; también me sorprendió mucho que ella lo hablara como si no tuviera importancia el hecho de no ser tomada con seriedad, aunque supongo que ha sido porque todas las relaciones que intenta que sean estables fueron violentas y terminaron dañando su autoestima. Me parece que necesita mucha ayuda psicológica e incluso psiquiátrica, primero, para revalorarse a sí misma y no depender de algu- na pareja estable ni meramente sexual para sentirse bien y no deprimirse, y segundo, porque es consciente de que está dañando también a su hija y a que a ésta, en el futuro, le cueste trabajo tener relaciones amorosas estables y sanas si no recibe también el apoyo psicológico de su madre'.

\section{Caso 2}

Varón de 14 años, estudiante de secundaria. Ingresa en urgencias llevado por su cuidadora, quien lo encontró inconsciente con quemaduras de segundo grado en la región pectoral, miembros superiores e inferiores, tras tocar dos cables eléctricos de su balcón. Presenta además delgadez extrema y la cuidadora comenta que había observado cambios en los ciclos de sueño, ingesta de comida, peso corporal y estado de ánimo, lo que le hace pensar que posiblemente intentó quitarse la vida. Es hijo único y tiene padres muy competitivos que solo dan importancia a tener logros y se centran más en sus actividades profesionales. De hecho, cuando ocurrió el incidente, se encontraban en el extranjero. Su conducta hacia el chico ha sido siempre de exigencia y descalificación cuando no cumple con sus expectativas.

El estudiante relata: 'de las cuatro historias clínicas que realicé, dos las trabajé con adolescentes y me resultó mucho más sencillo que con los adultos, tal vez por la cercanía de edades y porque en algunos momentos de nuestra vida pasamos por situaciones parecidas, como es el sentirnos rechazados o que no pertenecemos o encajamos en ningún lugar. Es triste ver que este tipo de muchachos busquen una salida tan fácil para ellos y difícil para la familia, que es la que al final se queda con la carga de la muerte o con las secuelas que quedan de estos errores, como en este caso, en que el desgaste lo padecen ambas partes. Conocer a fondo la historia de cada uno de ellos es extraordinario porque sabes qué hay más allá de la enfermedad y puedes comenzar a entender muchas cosas que no solo implican el puro conocimiento médico, te ayudan a relacionarte, a buscar la forma de meterte en la vida del paciente para descubrir cosas que a veces ni imaginamos y que pueden llevar a un buen o mal pronóstico, además de reconfortar al paciente y darle ánimos para seguir con su tratamiento, como debe ser, y así ganamos más porque nos hacemos buenos médicos. Es increíble la cantidad de aspectos que puede esconder una persona como paciente y lo que podemos descubrir nosotros mismos. Esta experiencia fue muy gratificante porque aunque quizá no volveré a ver ninguna de estas cuatro personas, me quedó la maravillosa sensación de que aunque 
médicamente tal vez no los ayudé, si lo hice escuchándolos y reflexionando con ellos varios aspectos que están implicados en la vida misma. Esto me apoyó mucho como médico en formación, como persona y, cuando esté al otro lado, como paciente'.

\section{Caso 3}

Mujer de 77 años, viuda, es costurera y trabaja en el hogar. Quince días antes de una caída presentaba déficit físico y cognitivo para realizar sus tareas cotidianas. Sufre una caída que le produce una factura de cadera izquierda, por lo que es ingresada, siendo difícil el tratamiento por el riesgo quirúrgico y la presencia de dolor continuo bastante intenso. Posteriormente se complica con un cuadro de neumonía, por lo que se la traslada a otro hospital.

La alumna comenta: 'al abordar esta historia clínica, me di cuenta de lo difícil que ha sido la elaboración de estas tres historias clínicas, pero lo mucho que ha cambiado desde que hice la primera hasta ahora. Fue la primera historia clínica médico-psicológica que realicé a una paciente de sexo femenino y el manejo es diferente. Siento que mi experiencia fue más difícil en esta ocasión, ya que en las dos anteriores eran pacientes masculinos que me abordaron y me empezaron a hablar. Con esta paciente fue más complicado al inicio de la entrevista y su nuera me preguntó el motivo de la misma. En todo momento le expliqué que era parte de mi formación como médico y me comentó que le daba mucho gusto, ya que muchas veces parece que a los médicos no les interesa. La paciente y su nuera, en el transcurso de la entrevista, fueron siendo más amables y me respondían a todas las cuestiones, por lo que lo hice en dos entrevistas para no cansarlas. Aunque la mayoría de los pacientes ingresados en el servicio son adultos mayores, no creo que les den el manejo correcto en cuanto a su estado psicológico. Muchos están abandonados y creen que su vida ya terminó: no les queda nada más por hacer'.

\section{Caso 4}

Varón de 54 años, soltero, diseñador de moda, con orientación homosexual y antecedente de abuso sexual en la infancia, que acude por sintomatología gastrointestinal caracterizada por dolor e intolerancia a la ingesta de grasas. A decir del paciente, se le diagnosticó un problema vesicular hace diez años, pero por temor a la cirugía decidió no intervenirse. Ahora ya se siente más confiado y quiere terminar con su malestar.
El alumno comenta: 'el inicio de la entrevista con Salvador fue un poco difícil, poco a poco tuve que ir ganándome su confianza. Conforme fue pasando el tiempo, siento que la relación se dio de mejor forma. Durante toda la entrevista percibí un ambiente de tristeza, de melancolía y una sensación de desamparo por su parte. Al final de la entrevista me sentí agotado, tanto física como emocionalmente. Saber que una persona había sufrido de la forma en que Salvador lo ha hecho me hizo sentir su tristeza y su propio desconsuelo.'

\section{Caso 5}

Mujer de 38 años, soltera, empleada de una papelería. Acude por presentar trabajo de parto; al respecto, menciona que finalmente se decidió a tener un hijo de un hombre del cual se había enamorado, pero éste, al enterarse que el feto tenía síndrome de Down, se alejó de ella. La paciente decidió hacerse cargo de su hijo con ayuda de sus padres y de Dios.

La estudiante comenta: 'Durante la entrevista me sentí muy a gusto con la paciente porque fue muy cooperadora, amable, no mostró pena o impaciencia ante la entrevista. También me sentí muy bien al realizarla porque me dio a entender que apreciaba el tiempo que le dedicaba, aunque a veces le parecía un poco tedioso. No fue muy difícil ganarme su confianza y eso me gustó porque temía que fuera complicado obtener respuestas a ciertas preguntas personales que podrían ser ofensivas para algunas personas. Creo que además de haber pasado un rato ameno en la entrevista, aprendí algo y eso es lo importante, siempre llevamos algo de cada experiencia de nuestra vida, aprendí cómo a algunas personas les cambia la vida de un día para otro, un tanto por el destino o suerte, y otro tanto, por decisión propia. Este cambio es muy fuerte; por ejemplo, en el caso de Ana María, de ser una mujer soltera sin compromisos a una madre soltera de un niño con discapacidad. Sin embargo, a pesar de este cambio radical, me enseñó que apoyándonos en diferentes cosas -cada uno sabrá cuáles- es posible salir adelante y siempre con optimismo ver lo bueno de la vida; así, las cosas son más fáciles'.

\section{Caso 6}

Mujer de 51 años, casada y con una licenciatura. Acude a urgencias por presentar disnea, cianosis, dolor ardoroso en la orofaringe, tos seca excesiva y cifras elevadas de tensión arterial, que se desencadenan por consumir pollo asado e ingerir un hueso que queda atravesado en la orofaringe. Refiere en 
su historia abuso sexual por parte de su padre a lo largo de diez años y de un vecino del barrio en que habitaba por cuatro años.

La estudiante relata: 'para ser la segunda entrevista, aún me sentía inexperta hablando, tanto que titubeaba cuando formulaba las preguntas. Al contrario del primer paciente, ésta tenía una forma muy peculiar de hablar, jamás me dijo una grosería y todo lo estructuraba con palabras formales, así que me esforcé en hablar de la misma forma para no incomodarla, lo cual fue una trabajo un tanto extraño. Como hemos visto en clase, para una buena relación médico-paciente, ambos deben entenderse con el vocabulario adecuado, pero en más de una ocasión tuve que detenerme en mi redacción y escuchar la historia con detalle, porque lo que ella me contaba era realmente impactante. Llegó un momento en que sentí un nudo en la garganta porque era demasiado fuerte lo que me decía, o quizás porque jamás había conocido en profundidad la vida de una paciente que había sido violada, por su padre y por un vecino, y que nadie hiciera nada, ni su propia madre. Mi cara nunca fue de asombro, ya que sentí que si hacia alguna gesticulación la paciente se ofendería y no iba a seguir contándome su situación. Así que me tomé mi papel muy en serio y la escuché. Al finalizar, la paciente lloró un poco y me dijo: "yo sé que siempre que cuento mi historia nadie me cree o piensan que siempre miento porque jamás dije nada o hice algo, pero quiero que entiendan que el amor a un padre es infinito y más si eres pequeña, no sabes que lo que te hace está mal; a mí, jamás nadie me lo dijo y yo lo respeto a él mucho; y con el vecino, creí que todos los hombres que lo hicieran sería normal, así que no guardé rencores en mi vida». Salí de la entrevista impactada, pero antes de concluir le di las gracias por permitirme realizar la entrevista y por sincerarse conmigo. Le deseé su pronta recuperación y me despedí con la mano, pero ella se acercó a mí para darme un beso en la mejilla y me dijo: «gracias a ti por escucharme, espero haber sido de ayuda en tu tarea y échale muchas ganas, no te pongas nerviosa»'

\section{Discusión}

En los seis ejemplos descritos se observan varios de los aspectos relacionados con la investigación realizada al respecto, siendo abordado tanto por técnicas cualitativas como algunas cuantitativas. En cada uno de los seis casos expuestos, los estudiantes presentan reacciones frecuentes y parecidas a las halladas en estudios previos con las respuestas de los estudiantes de medicina. Por ejemplo, muchos de ellos, principalmente mujeres, presentaron temor, inseguridad y ansiedad para relacionarse con el paciente, e incluso incomodidad al hablar sobre aspectos íntimos [12].

Por otro lado, el tercer y el cuarto caso hacen referencia a la dificultad de ganarse la confianza de los pacientes y abordarlos en calidad de estudiante, en particular cuando se trata de una adulta mayor y además se tiene contacto con la familia; esto les permite conocer su entorno social y familiar, comprendiendo que la enfermedad ocurre de manera diferente en cada persona y que adquiere consecuencias y significados propios en cada caso [6], como se muestra en el segundo ejemplo.

Se observa también que la mayoría de las veces los pacientes están de acuerdo en ser entrevistados por estudiantes, e incluso reconocen que les dedican más atención, aclaran sus dudas y se sienten agradecidos con ellos por escucharlos [7], como se observa en los últimos casos.

La realización de este tipo de historia clínica por parte de los estudiantes de medicina al inicio de su formación clínica es una experiencia vivencial directa que va más allá de la teoría médico-científica, ya que deja un aprendizaje con un importante impacto emocional importante, pues le permite el contacto directo con el ser humano enfermo y no solamente con la enfermedad, lo que favorece el desarrollo y consolidación de la relación médico-paciente. Estos conceptos fueron fortalecidos por este grupo de alumnos, lo cual se demostró cualitativamente en esta investigación. Los resultados de esta experiencia reflejan que, para los participantes, fue una oportunidad efectiva de aprendizaje que podría ser parte de la formación de estudiantes de medicina.

Para futuras investigaciones se podrían realizar entrevistas a los estudiantes con preguntas dirigidas hacia cómo se sienten previamente a encontrarse con un paciente, cómo creen que lo perciben los pacientes y qué esperan de ellos, haciendo una comparación entre un antes y después del encuentro, así como aspectos en los que creen que pueden mejorar.

\section{Bibliografía}

1. Martínez R. The nature of illness experience: a course on boundaries. Theor Med Bioeth 2002; 23: 259-69.

2. Dornan T, Bundy C. What can experience add to early medical education? Consensus survey. BMJ 2004; 329: 834.

3. Hicks LK, Lin Y, Robertson DW, Robinson DL, Woodrow SI. Understanding the clinical dilemmas that shape medical students' ethical development: questionnaire survey and focus group study. BMJ 2001; 322: 709-10.

4. Yedidia MJ, Gillespie CC, Kachur E, Schwartz MD, Ockene J, Chepaitis AE, et al. Effect of communication: training on medical student performance. JAMA 2003; 290: 1157-65 
5. Tena-Tamayo C. La comunicación humana en la relación médico-paciente. 2 ed. México DF: Prado; 2007.

6. Ortiz A, Beca JP, Salas S, Browne F, Salas C. Acompañamiento del enfermo: una experiencia de aprendizaje sobre significado de la enfermedad. Rev Med Chile 2008; 136: 304-9.

7. Beca JP, Browne F, Valdebenito C, Bataszew A, Martínez MJ. Relación estudiante-enfermo: visión del paciente. Rev Med Chile 2006; 134: 955-9.

8. Spencer J, Blackmore D, Heard S, McCrorie P, McHaffie D, Scherpbier A, et al. Patient-oriented learning: a review of the role of the patient in the education of medical students. Med Educ 2000; 34: 851-7.

9. Chipp E, Stoneley S, Cooper K. Clinical placements for medical students: factors affecting patients' involvement in medical education. Med Teach 2004; 26: 114-9.
10. Glasser M, Bazuin CH. Patients' views of the medical education setting. J Med Educ 1985; 60: 745-56.

11. Prislin M, Morrison E, Giglio M, Truong P, Radecki S. Patients' perceptions of medical students in a longitudinal family medicine clerkship. Fam Med 2001; 33: 187-91.

12. Beca JP, Browne F, Repetto P, Ortiz A, Salas C. Relación estudiante de medicina-enfermo: visión de los estudiantes. Rev Med Chile 2007; 135: 1503-9.

13. Kiyohara LY, Kayano LK, Kobayashi ML, Alessi MS, Yamamoto MU, Yunes-Filho PR, et al. The patient-physician interactions as seen by undergraduate medical students'. Sao Paulo Med J 2001; 119: 97-100.

14. Rodríguez-Gómez G, Gil-Flores J, García-Jiménez E. Metodología de la investigación cualitativa. Málaga: Aljibe; 1996. 